OPEN ACCESS

Edited by: Jerome Collemare,

Westerdijk Fungal Biodiversity Institute, Netherlands

Reviewed by:

JinRong Xu,

Purdue University, United States

Ingo Bauer,

Innsbruck Medical University, Austria

${ }^{*}$ Correspondence:

Wenxing Liang

wliang1@qau.edu.cn

Specialty section:

This article was submitted to

Fungi and Their Interactions,

a section of the journal

Frontiers in Microbiology

Received: 27 April 2020

Accepted: 13 July 2020

Published: 29 July 2020

Citation:

Zhang N, Yang Z, Zhang Z and Liang W (2020) BCRPD3-Mediated Histone Deacetylation Is Involved

in Growth and Pathogenicity

of Botrytis cinerea.

Front. Microbiol. 11:1832

doi: 10.3389/fmich.2020.01832

\section{BcRPD3-Mediated Histone} Deacetylation Is Involved in Growth and Pathogenicity of Botrytis cinerea

\author{
Ning Zhang', Zhenzhou Yang ${ }^{2}$, Zhonghua Zhang ${ }^{3}$ and Wenxing Liang ${ }^{2 *}$ \\ ${ }^{1}$ Tobacco Research Institute, Chinese Academy of Agricultural Sciences, Qingdao, China, ${ }^{2}$ Key Lab of Integrated Crop Pest \\ Management of Shandong, College of Plant Health and Medicine, Qingdao Agricultural University, Qingdao, China, ${ }^{3}$ Institute \\ of Vegetables and Flowers, Chinese Academy of Agricultural Sciences, Beijing, China
}

Histone deacetylase activity plays an important role in transcriptional repression. Botrytis cinerea is an important necrotrophic fungal pathogen distributed worldwide and parasites a wide range of hosts. However, the molecular mechanisms of how B. cinerea regulates growth and host infection remain largely unknown. Here, the function of BcRPD3, a histone deacetylase of $B$. cinerea, was investigated. Overexpression of the $B C R P D 3$ gene resulted in significantly decreased acetylation levels of histone $\mathrm{H} 3$ and H4. The BCRPD3 overexpression strains showed slightly delayed vegetative growth, dramatically impaired infection structure formation, oxidative stress response, and virulence. RNA-Seq analysis revealed that enzymatic activity related genes, including 9 genes reported to function as virulence factors, were downregulated in BCRPD3 overexpression strain. Chromatin immunoprecipitation followed by aPCR confirmed the enrichment of BcRPD3 and H3Kac at the promoter regions of these nine genes. These observations indicated that BcRPD3 regulated the transcription of enzymatic activity related genes by controlling the acetylation level of histones, thereby affecting the vegetative growth, infection structure formation, oxidative stress response, and virulence of $B$. cinerea.

\section{Keywords: Botrytis cinerea, histone acetylation, deacetylase, pathogenicity, RNA-seq}

\section{INTRODUCTION}

Chromatin remodeling through histone acetylation is an important epigenetic mechanism controlling gene transcription. Histone hyperacetylation results in a loose chromatin structure and transcription activation, while hypoacetylation of histones leads to transcriptional repression by chromatin condensation. The acetylation of histones is antagonistically controlled by histone acetyltransferases (HATs) and histone deacetylases (HDACs) (Lee and Workman, 2007). Characterizing functions of HATs and HDACs is an important way to clarify the roles of histone acetylation.

HDACs are a family of enzymes that reverse lysine acetylation catalyzed by HATs through deacetylation of lysine residues on histones. Except for its important roles in the epigenetic regulation of gene expression, recent studies have uncovered a plethora of non-histone targets 
of previously known HATs and HDACs, including transcription factors, signaling proteins and other proteins (Emiliani et al., 1998; Bernstein et al., 2000; Yang and Seto, 2008). The acetylation events reversed by HDACs play critical roles for a mass of cellular events, such as protein folding, energy utilization, and cellular metabolism (Imai and Guarente, 2010; Guan and Xiong, 2011). This gene family is conserved in different organisms, comprising of four classes according to sequence homology. Class I, II and IV enzymes are zinc-dependent HDACs. Class III consists of Sir2 or sirtuin family which are $\mathrm{NAD}^{+}$dependent (Gregoretti et al., 2004; Jeon et al., 2014).

RPD3 is the founding member of the Class I HDACs in Saccharomyces cerevisiae (Rundlett et al., 1996; Kurdistani and Grunstein, 2003), regulating gene expression through deacetylation of the histones H3 and H4 (Bernstein et al., 2000; Sabet et al., 2004). The genes targeted by RPD3 have diverse functions, including osmotic-stress responsive genes, DNA damage inducible genes, and those involved in determination of replication origin firing (Vogelauer et al., 2002; De Nadal et al., 2004; Sharma et al., 2007).

Filamentous fungi possess RPD3 homologs, playing essential roles in the fungal growth, development and virulence (Lechner et al., 2000; Trojer et al., 2003; Carrozza et al., 2005). For instance, depletion of RpdA in Aspergillus nidulans leads to a pronounced reduction of growth and sporulation of the fungus (Tribus et al., 2010). In Aspergillus fumigatus, RpdA is essential for virulence and loss of RpdA activity results in a lethal phenotype (Bauer et al., 2016, 2019). Hda1 in Ustilago maydis, with homology to the yeast RPD3, regulates a subset of genes essential for mature teliospore formation and represses fungal penetration into host epidermal cells (Reichmann et al., 2002; Torreblanca et al., 2003). In Beauveria bassiana, RPD3 plays essential roles in regulating transcription of almost all genes in the central development pathway (Cai et al., 2018). MoRPD3 disruption in Magnaporthe oryzae is lethal (Izawa et al., 2009). Botrytis cinerea, known as gray mold, can infect over 200 different plant species and is among the most important postharvest fungal pathogens worldwide (Dean et al., 2012). However, there is a lack of reports on the role of RPD3 in $B$. cinerea and its impact on growth and pathogenicity.

Here we investigated the function of BcRPD3 as a histone deacetylase and its impact on fungal development and pathogenesis in $B$. cinerea. Our data revealed that BcRPD3 is involved in the deacetylation of histone $\mathrm{H} 3$ and $\mathrm{H} 4$. Overexpression of $B C R P D 3$ exhibited a relatively limited effect on vegetative growth, but dramatically affected infection structure formation, oxidative stress response, and virulence in B. cinerea. RNA-seq analysis revealed that enzymatic activity related genes were significantly downregulated in $B c R P D 3$ overexpression strain compared with wild type, including 9 genes reported to function as virulence factors in $B$. cinerea. Moreover, Chromatin immunoprecipitation followed by qPCR demonstrated that BcRPD3 is recruited to promoter regions of these nine genes, which leads to a decrease of $\mathrm{H} 3 \mathrm{Kac}$ abundance at these regions, suggesting an important transcriptional regulatory role of $\mathrm{BcRPD} 3$ and $\mathrm{H} 3 \mathrm{Kac}$ in B. cinerea.

\section{MATERIALS AND METHODS}

\section{Strains and Culture Conditions}

Wild type B05.10 of B. cinerea was used for transformation experiments in this study. All strains were maintained on PDA plates ( $2 \%$ dextrose, $20 \%$ potato, and $1.5 \%$ agar) or in YPD medium (2\% peptone, $1 \%$ yeast extract, and $2 \%$ glucose). Mycelial growth of the tested strains was measured after 3 days cultivated on PDA plates in $25^{\circ} \mathrm{C}$. The conidia number were counted after 10 days incubated on PDA plates. Conidial germination assays were determined as previously described (Liu Y. et al., 2019). Briefly, fresh conidia of wild type, BcRPD3-1, and VRPP3 after 10 days incubation on PDA plates were harvested in sterilized water and adjusted to the concentration of $2.5 \times 10^{5}$ conidia/ml in PDB (liquid PDA). Twenty microliter of the conidial suspension were dropped onto coverslips and incubated in a moist chamber with a temperature of $25^{\circ} \mathrm{C}$ and germination rates were determined with over 100 conidia per replicate.

\section{Generation of BcRPD3 and BCRPD3-VRPP Overexpression Strains}

To generate $B c R P D 3$ and $B c R P D 3-V R P P$ overexpression construct, CDS of BcRPD3 and BcRPD3-VRPP was cloned into $N c o$ I digested pOPT-GFP vector that contains oliC promoter, niaD terminator, hygromycin phosphotransferase and optimized C-terminal eGFP sequence (Leroch et al., 2011), respectively. The primers used for vector construction are listed in Supplementary Table S1. The constructed plasmids were then transformed into the B05.10 strain using protoplast transformation of $B$. cinerea (Gronover et al., 2001). The resulting transformants were selected by $100 \mu \mathrm{g} / \mathrm{ml}$ hygromycin B and further confirmed by western blot using anti-GFP antibody.

\section{Assays of Pathogenicity, Infection Cushion Formation, and Sensitivity to $\mathrm{H}_{2} \mathrm{O}_{2}$}

Pathogenicity assays were measured as described previously (Yang et al., 2018). In brief, fresh harvested spores of the tested strains were adjusted into same concentration of $5 \times 10^{5}$ conidia/ml in $10 \mathrm{mM}$ glucose, dropped on 3 weeks old tomato leaves and incubated at $25^{\circ} \mathrm{C}$ for 3 days. Then the lesion diameters caused by different strains with at least five repeats were measured. For infection cushion formation assays, five $\mathrm{mm}$ diameter plugs of tested strains cultivated on PDA plates for 3 days were placed on the surfaces of the glass slides and incubated in a moistened chamber at $25^{\circ} \mathrm{C}$. After 24,48 , and $60 \mathrm{~h}$ post incubation, total number of infection cushions in at least five randomly selected view areas per replicate was examined respectively. To measure sensitivity to $\mathrm{H}_{2} \mathrm{O}_{2}$, samesized mycelium plugs of different strains were incubated on PDA plates for 3 days supplemented with $0.2 \% \mathrm{H}_{2} \mathrm{O}_{2}$ at $25^{\circ} \mathrm{C}$ and the mycelial growth was then measured.

\section{RNA Sequencing}

The mycelia of wild type B05.10 and BcRPD3-1 strain with three biological replicates were harvested after growth in YPD 
medium with shaking at $120 \mathrm{rpm}$ for $10 \mathrm{~h}$ in $25^{\circ} \mathrm{C}$. Total RNA was extracted using the TRIzol reagent according to the instructions of manufacturer. RNA-seq data were analyzed as previously described (Rodenburg et al., 2018). Briefly, Cutadapt (v1.16) software was used to filter the sequencing data. Reference genome index was built by Bowtie2(2.2.6) and the filtered reads were mapping to the reference genome using Tophat2(2.0.14) The mapping statistics were shown in Supplementary Table S2. HTSeq(0.9.1) statistics was used to compare the Read Count values on each gene as the original expression of the gene, and then FPKM was used to standardize the expression. $\operatorname{DESeq}(1.30 .0)$ was used to analyze the genes of difference expression with screened conditions as follows: an absolute $\log _{2}$ value $>1$ and $P<0.05$. All the detected genes were shown in Supplementary Table S3. At the same time, we used $\mathrm{R}$ language Pheatmap(1.0.8) software package to perform bidirectional clustering analysis of all different genes of samples. GO categories represented by up- and down-regulated genes were demonstrated using g:Profiler toolset (Raudvere et al., 2019).

\section{Fluorescent Real-Time qPCR}

For qRT-PCR assessment of BcRPD3 expression during host infection, $50 \mu \mathrm{L}$ droplets of spore suspension $\left(2 \times 10^{6}\right.$ conidia $/ \mathrm{mL}$ ) of B05.10 were inoculated on the intact leaves of tomato and incubated in $22^{\circ} \mathrm{C}$. At $0,6,12,24,36$, and 48 hpi, inoculated leaf samples were collected for RNA extraction. The mycelium was collected after growth in YPD medium with shaking at $180 \mathrm{rpm}$ for $12 \mathrm{~h}$ in $25^{\circ} \mathrm{C}$. For validation of RNA-seq data, three batches of biological repeats of wild type and BcRPD31 were independently collected. RNA was extracted and reverse transcribed using All-In-One RT MasterMix (abm). qRT-PCR was performed using M5 HiPer SYBR Premix EsTaq (Mei5bio). The transcript abundance of candidate genes were calculated using the $2^{-\triangle C t}$ method. All primers used for qRT-qPCR were listed in Supplementary Table S1.

\section{Western Blot Assay}

The mycelia of the tested strains were grown in YPD at $25^{\circ} \mathrm{C}$ for $12 \mathrm{~h}$ in a shaker. The nuclear proteins were then extracted using Nuclear Protein Extraction Kit (R0050, Solarbio). The obtained proteins were separated by SDS-PAGE and immunoblotted using anti-GFP antibody (ab290, Abcam), anti-H3 antibody (ab1791, Abcam), anti-H4 antibody (07-108, Millipore), antiH3Kac antibody (06-599, Millipore), anti-H4Kac antibody (06598, Millipore), anti-H3K9ac antibody (PTM-156, PTM BIO), anti-H3K14ac antibody (PTM-157, PTM BIO), anti-H3K18ac antibody (PTM-158, PTM BIO), and anti-H3K27ac antibody (PTM-160, PTM BIO).

\section{ChIP-qPCR Analysis}

ChIP was performed according to described methods (Liu Z. et al., 2019). Briefly, The mycelia of wild type, GFP containing wild type and BcRPD3-1 strain were harvested after growth in YPD medium with shaking at $180 \mathrm{rpm}$ for $12 \mathrm{~h}$ in $25^{\circ} \mathrm{C}$. After fresh mycelia had developed, mycelia were cross-linked with $1 \%$ formaldehyde (PBS) gently shaking for $25 \mathrm{~min}$ and then stopped with glycine with a final concentration of $125 \mathrm{mM}$ for another incubation of $10 \mathrm{~min}$. After cleaning with sterile water for several times, the cultures were frozen and ground with liquid nitrogen. The powder was re-suspended in the lysis buffer $(250 \mathrm{mM}$ HEPES pH 7.5, 1 mM EDTA, 150 mM NaCl, 10 mM DTT, 0.1\% DeoxyCholate, and 1\% Triton) and protease inhibitor cocktail (Roche) with a mycelia/buffer ratio as $0.5 \mathrm{~g} / 2 \mathrm{ml}$. The DNA was sheared into $\sim 500$ bp fragments using sonicator (Scientz-650E, $35-60 \%$ amplitude, ultrasonication for $10 \mathrm{~s}$ and stop for $20 \mathrm{~s}$, 15 times). The supernatant was diluted after centrifugation with ChIP dilution buffer (1.1\% Triton X-100, 16.7 mM Tris-HCl pH 8.0, $1.2 \mathrm{mM}$ EDTA, $167 \mathrm{mM} \mathrm{NaCl}$ ). Immunoprecipitation was conducted using $5 \mu \mathrm{l}$ anti-GFP antibody (ab290, Abcam) or $5 \mu \mathrm{l}$ anti-H3Kac antibody (06-599, Millipore) together with protein A agarose (Roche) overnight at $4^{\circ} \mathrm{C}$. After separation, beads were washed orderly by low-salt wash buffer ( $150 \mathrm{mM} \mathrm{NaCl}, 0.2 \%$ SDS, 20 mM Tris-HCl PH 8.0, 2 mM EDTA, 0.5\% TritonX-100), high salt wash buffer (500 mM NaCl, 2 mM EDTA, $20 \mathrm{mM}$ Tris- $\mathrm{HCl}$ PH 8.0, 0.2\% SDS, 0.5\% TritonX-100), LiCl wash buffer $(0.25 \mathrm{M}$ $\mathrm{LiCl}, 1 \%$ Non-idet P-40, 1\% sodium deoxycholate, 1 mM EDTA, $10 \mathrm{mM}$ Tris- $\mathrm{HCl} \mathrm{pH} \mathrm{8.0),} \mathrm{and} \mathrm{TE} \mathrm{buffer.} \mathrm{DNA} \mathrm{bound} \mathrm{to} \mathrm{the} \mathrm{beads}$ was then eluted and precipitated. ChIP-qPCR was independently repeated three times. Relative enrichment values were calculated by dividing the immunoprecipitated DNA by the input DNA. Primers using for ChIP-qPCR were designed near putative TSS (transcription start site) and listed in Supplementary Table S1.

\section{RESULTS}

\section{Effect of BcRPD3 Overexpression on $\mathrm{H} 3$ and H4 Deacetylation}

To identify RPD3 in $B$. cinerea, we used the amino acid sequence of yeast RPD3 protein as query to search against the $B$. cinerea database at EnsemblFungi. As a result, the gene BcRPD3 (Ensembl: Bcin05g02590) was identified, which encodes a 633 amino acid protein with a DNA sequence of 3234 bp with three introns. A phylogenetic tree of fungal RPD3 orthologs was constructed (Figure 1A). Fungal orthologs of RPD3 are found among necrotrophic fungi (Botrytis cinerea, Colletotrichum incanum, Aspergillus fumigatus), biotrophic fungi (Blumeria graminis, Melampsora larici-populina, Sporisorium reilianum, Bipolaris zeicola, Magnaporthe oryzae, Fusarium graminearum, Ustilago maydis), and yeasts (Cyberlindnera jadinii, Kluyveromyces marxianus, Zygosaccharomyces bailii, Saccharomyces cerevisiae). These orthologs clustered into three distinct groups: Pezizomycotina, Saccharomycotina, and Basidiomycota, and all orthologs contained a HDA deacetylase domain (Figure 1B).

qRT-PCR was used to assess the expression of BcRPD3 during plant infection and vegetative growth phase (mycelium). As shown in Supplementary Figure S1, expression of BcRPD3 decreased during infectious growth stages, while in mycelium, the expression was elevated and significantly higher than in conidia $(0 \mathrm{~h})$. This transcription pattern implicated that BcRPD3 was more active during vegetative growth phase than during plant infectious stages in B. cinerea, indicating that $B c R P D 3$ 


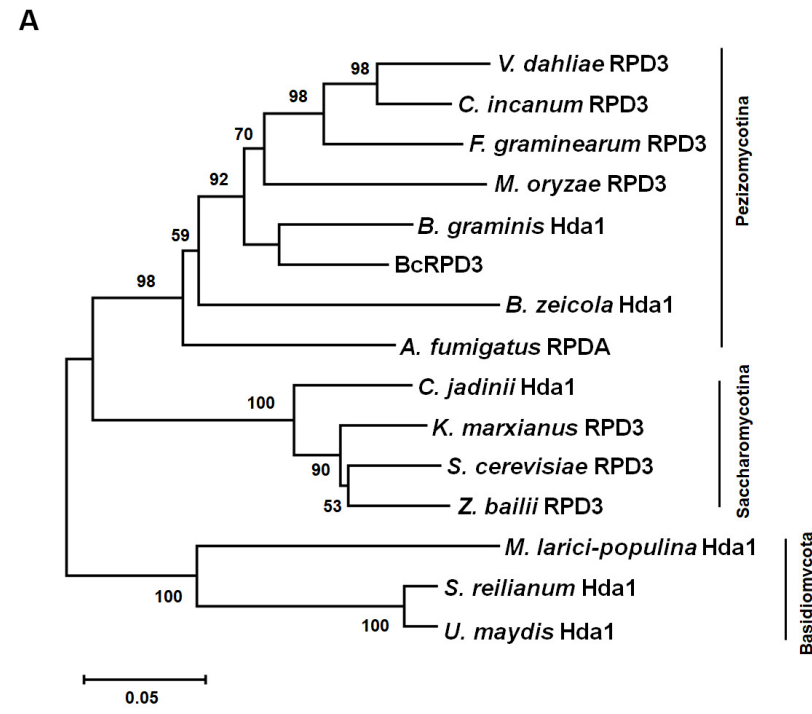

C
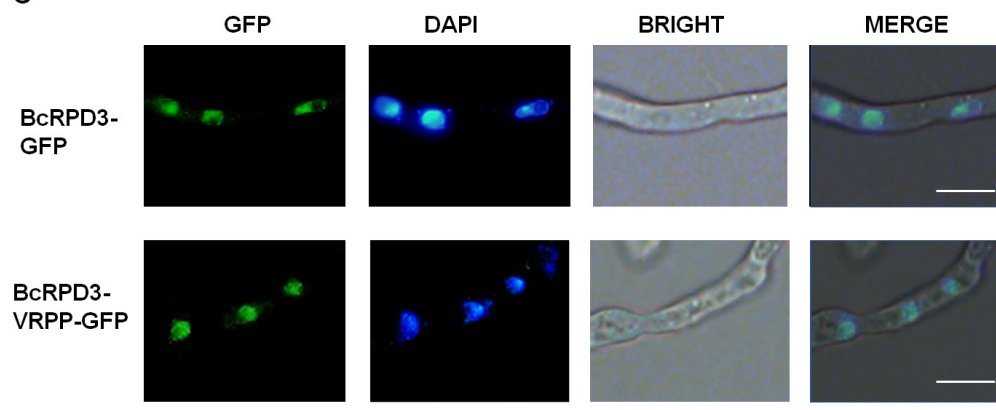

B

\begin{tabular}{|c|c|c|}
\hline V. dahliae RPD3 & HDA & $1-779$ \\
\hline C. incanum RPD3 & HDA & $1-680$ \\
\hline F. graminearum $\mathrm{RPD} 3$ & HDA & $1-649$ \\
\hline M. oryzae RPD3 & HDA & $1-659$ \\
\hline B. graminis Hda1 & HDA & $1-606$ \\
\hline BcRPD3 & HDA & $1-633$ \\
\hline B. zeicola Hda1 & HDA & $1-648$ \\
\hline A. fumigatus RPDA & HDA & $1-688$ \\
\hline C. jadinii Hda1 & HDA & $1-436$ \\
\hline K. marxianus RPD3 & HDA & $1-432$ \\
\hline S. cerevisiae RPD3 & HDA & $1-433$ \\
\hline Z. bailii RPD3 & HDA & $1-433$ \\
\hline M. larici-populina Hda1 & HDA & $1-388$ \\
\hline S. reilianum Hda1 & HDA & $1-610$ \\
\hline U. maydis Hda1 & HDA & $1-569$ \\
\hline
\end{tabular}

D Anti-H3Kac Anti-H3K9ac

Anti-H3K14ac

Anti-H3K18ac

Anti-H3K27ac

Anti-H4Kac

Anti-H4

Anti-H3

FIGURE 1 | Classification and localization of BCRPD3. (A) Phylogenetic tree of BCRPD3 based on a neighbor-joining analysis using MEGA-X. The orthologs are from V. dahliae RPD3 (Ensembl: PNH36023), C. incanum RPD3 (Ensembl: KZL80576), F. graminearum RPD3 (Ensembl: ESU06006), M. oryzae RPD3 (Ensembl: MGG_05857TO), B. graminis Hda1 (Ensembl: BLGH_01902), B. zeicola HDA1 (Ensembl: EUC28153), A. fumigatus RPDA (Ensembl: OXN03576), C. jadinii Hda1 (Ensembl: ODV71565), K. marxianus RPD3 (Ensembl: BAO39086), S. cerevisiae RPD3 (Ensembl: KZV08295), Z. bailii RPD3 (Ensembl: SJM84961), M. Iarici-populina Hda1 (Ensembl: EGG09787), S. reilianum Hda1 (Ensembl: CBQ72606), and U. maydis HDA1 (Ensembl: KIS69526). (B) Schematic representation of different RPD3 orthologs including their catalytic domains. HDA, histone deacetylase domain. (C) Mycelia images for subcellular localization of the GFP-tagged BcRPD3 and BcRPD3-VRPP. Bars $=10 \mu \mathrm{m}$. (D) Western blotting for the expression levels of histone $\mathrm{H} 4, \mathrm{H} 3$ and acetylation levels of H4, $\mathrm{H} 3$ and its Kac sites in wild type B05.10, VRPP-3, and BCRPD3-1 strains.

may play different roles during multiple growth conditions and developmental stages.

To elucidate the functions of $B c R P D 3$, we first tried to delete the $B C R P D 3$ gene. However, despite numerous attempts (over 200 transformants), we failed to gain BcRPD3 knock out mutants, indicating that $B c R P D 3$ plays an essential role for the survival of $B$. cinerea. Alternatively, we generated overexpression strains of $B C R P D 3$ with a oliC promoter and C-terminal GFP in B. cinerea. Previous studies showed that swapping the amino acids AGG in HDAC1 (human homolog of BcRPD3) with VRPP which is unique amino acid sequence in class II HDACs, resulted in a loss-of-function mutation of HDAC activity (Wei et al., 2017). We used the amino acid sequences of class I and class II HDACs in human to search against the $B$. cinerea database. As a result, $\mathrm{BcRPD} 3$ and $\mathrm{BcH}$ os 2 (Bcin01g03610) were identified as class I HDACs, while BcHda1 (Bcin15g02130) and BcHos1 (Bcin12g01310) was identified as class II HDACs in B. cinerea (Supplementary Figure S2A). Accordingly, we generated $B C R P D 3-V R P P$ overexpression strains to serve as a silencing transformant of impaired deacetylase activity. A total of 3 transformants were obtained for BcRPD3 and $B c R P D 3-V R P P$ overexpression, respectively. We used qRTPCR to assess the expression level of $B c R P D 3$ in different strains and the results indicated that $B C R P D 3$ transcript showed marked up-regulation in overexpression lines compared with B05.10 (Supplementary Figure S2B). In addition, these 3 transformants of each group had the same phenotypes and western analysis with anti-GFP showed indistinguishable protein expression levels (Supplementary Figure S2C). Therefore, only data for transformants BcRPD3-GFP-1 (BcRPD3-1) and BcRPD3-GFPVRPP-3 (VRPP-3) were presented below.

Fluorescence microscopy was used to observe the transformed mycelia after growth on PDA plates for 2 days. Both of the green signals of the GFP-tagged BcRPD3 and BcRPD3-VRPP 
overlapped with nuclei stained with DAPI (Figure 1C), indicating BcRPD3 and BcRPD3-VRPP are both mainly located in nucleus. Since RPD3 orthologs reportedly target the acetylation of histones in yeast and filamentous fungus A. nidulans (Rundlett et al., 1998; Graessle et al., 2000; Chang and Pillus, 2009; Tribus et al., 2010), protein extracts of wild type B05.10, strain BcRPD3-1 and VRPP-3 were subjected to immunoblotting using acetyl-histone $\mathrm{H} 3, \mathrm{H} 4$ antibodies. The results showed that the acetylation levels of both $\mathrm{H} 3$ and $\mathrm{H} 4$ significantly decreased in strain BcRPD3-1 compared with wild type, while BcRPD3-VRPP was hardly to detect any significant HDAC activity (Figure 1D and Supplementary Figure S2D). Furthermore, strain BcRPD31 exhibited hypoacetylation of histone $\mathrm{H} 3$ at $\mathrm{K} 9, \mathrm{~K} 14$, and $\mathrm{K} 27$ in comparison to the blots of wild type and strain VRPP-3 (Figure 1D). The altered Kac events indicated an ability for BcRPD3 to deacetylate the Kac sites of $\mathrm{H} 3$ and $\mathrm{H} 4$ in B. cinerea.

\section{Impact of BcRPD3 Overexpression on Radial Growth, Sporulation, and Conidial Germination}

To determine whether BcRPD3 is involved in B. cinerea growth, we examined mycelial growth of wild type, BcRPD3-1, and VRPP-3 strains. Our data demonstrated that strain BcRPD31 exhibited significant but limited radial growth decrease (around 25\%) as compared with the wild type, while strain VRPP-3 didn't show any significant difference with wild type (Figures 2A,B). To test whether BCRPD3 played a role in $B$. cinerea conidiation, we determined conidiation ability of tested strains after 10 days inoculated on PDA plates. Our results demonstrated that conidiation of the BcRPD3-1 strain was almost same as wild type and VRPP-3 strains (Figures 2C,D). To determine the viability of conidia produced by the BCRPD3 overexpression, we observed conidial germination of the conidia harvested from 12-day incubated plates. Our data indicated that overexpression of $B C R P D 3$ delayed conidial germination on glass slides (Figure 2E). The above data indicated that overexpression of $B C R P D 3$ slightly affected $B$. cinerea vegetative growth and germination.

\section{BcRPD3 Overexpression Affects Virulence, Infection Structure Formation, and Oxidative Stress Response of}

\section{B. cinerea}

To investigate the function of $B c R P D 3$ in $B$. cinerea pathogenicity, an infection assay on tomato leaves was performed. Two days after inoculation, spreading lesions caused by BcRPD31 strain was significantly less than wild type and VRPP3 strain, which is almost reaching 50\% (Figures 3A,B). To evaluate the effect of $B c R P D 3$ on infection structure formation, we observed infection cushion formation of the tested strains. Our data demonstrated that compared with wild type and VRPP-3 strain, BcRPD3-1 strain significantly formed less infection cushions after 24-h incubation and resumed after 48-h incubation on glass slides (Figures 3C,D), indicating that formation of infection cushions was delayed by $B c R P D 3$ overexpression.
Since sensitivity to oxidative stress is associated with loss of pathogenicity of B. cinerea (Yang et al., 2018), we investigated the sensitivity of hyphae of tested strains to oxidative stress. After growth on PDA plates supplemented with $0.2 \%$ $\mathrm{H}_{2} \mathrm{O}_{2}$ for 3 days, the relative growth reduction of colony diameter achieved by BcRPD3-1 strain was almost twice as wild type and VRPP-3 strains (Figures 3E,F). Taken together, these data demonstrated that overexpression of BcRPD3 has negative effects on mediating infection cushion formation and resistance to oxygen stress, which may be partially responsible for the virulence-attenuation of the pathogen overexpressing BcRPD3.

\section{BcRPD3 Overexpression Downregulated Expression of Enzymatic Activity Related Genes}

Previous studies have demonstrated that RPD3 is localized in the nucleus and can catalyze deacetylation of histone $\mathrm{H} 3$ and $\mathrm{H} 4$ in S. cerevisiae and filamentous fungus A. nidulans (Rundlett et al., 1998; Graessle et al., 2000; Chang and Pillus, 2009; Tribus et al., 2010). As shown in Figure 1D, BcRPD3 overexpression significantly reduced the acetylation levels of $\mathrm{H} 3$ and H4. Therefore, we performed an RNA-Seq analysis to detect genes that might exhibit changes in regulation affected by BcRPD3 in B. cinerea. Three biological replicates with mRNA isolated from wild type B05.10 and BcRPD31 strain were performed. As a result, 548 down- and 355 upregulated (fold change $>2, P<0.05$ ) genes were identified in the strain BcRPD3-1 compared with B05.10 (Figure 4A and Supplementary Table S3).

Given the function of histone deacetylases in gene repression, we supposed that the target genes of BcRPD3 were likely to be among the downregulated genes. Functional annotation of Gene Ontology (GO) analysis of those downregulated genes revealed that genes involved in enzymatic activity, including oxidoreductase, cofactor binding, glycosyl hydrolase, and catalytic activity were significantly enriched (Figure $4 B$ ). Nine downregulated genes (Figure 4C) from representative categories were selected for experimental validation. These 9 genes were reported to be involved in pathogenicity of $B$. cinerea, including oxidoreductase activity-related $B c A o x$ (alternative oxidase) (Lin et al., 2019), cofactor biding-related BcBoa3 and BcBoa4 (botcinic acid biosynthetic genes) (Dalmais et al., 2011), glycosyl hydrolase activity-related BcPg1 (endo-polygalacturonase) (Have et al., 1998), and BcAra1 (endo-arabinanase) (Nafisi et al., 2014), catalytic activity-related BcBot1, BcBot5 (botrydial biosynthetic genes) (Dalmais et al., 2011), and BcPck1 (phosphoenolpyruvate carboxykinase) (Liu et al., 2018), and BcSpl1 (cerato-platanin family protein) (Frías et al., 2011). qRT-PCR analysis indicated that all of these 9 genes were indeed downregulated in strain BcRPD3-1 compared with B05.10 (Figure 4D). Besides, it is noteworthy that a large proportion of $B c B o a$ and $B c B o t$ family genes regulating synthesis of two groups of non-specific phytotoxins, botrydial and botcinic acid, were dramatically downregulated (Figure 4E), which is in accord with repeated reports that interference with KDAC function 


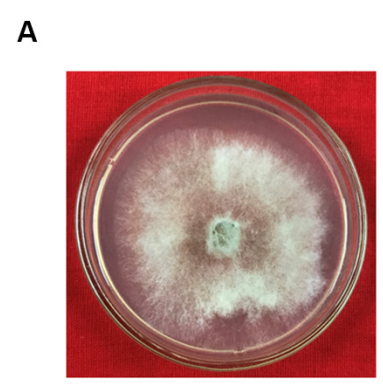

B05.10

C

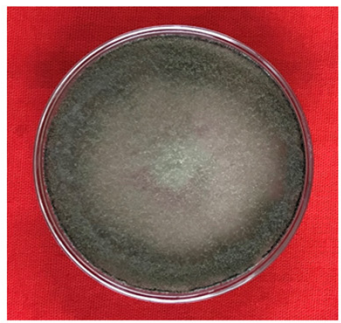

B05.10

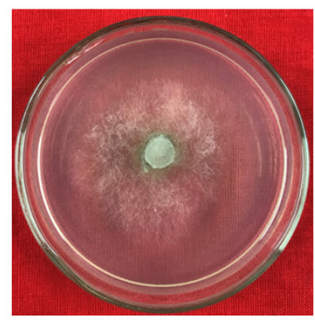

BcRPD3-1

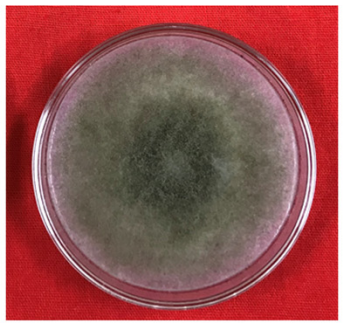

BcRPD3-1

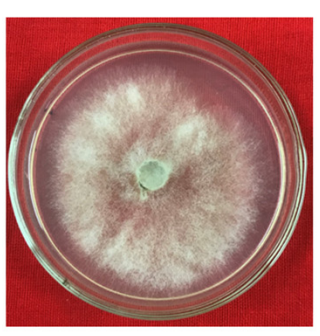

VRPP-3

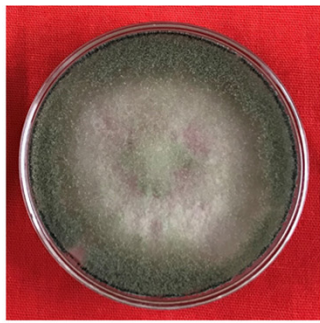

VRPP-3
B

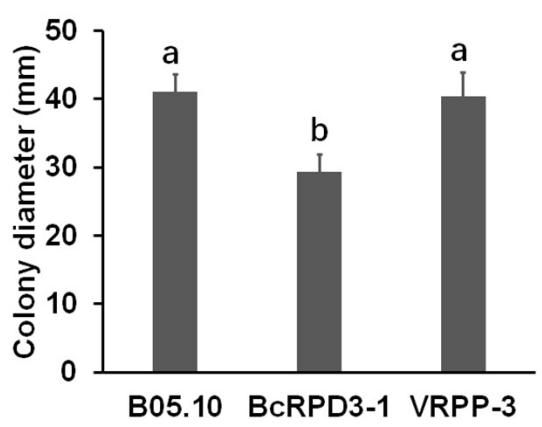

D

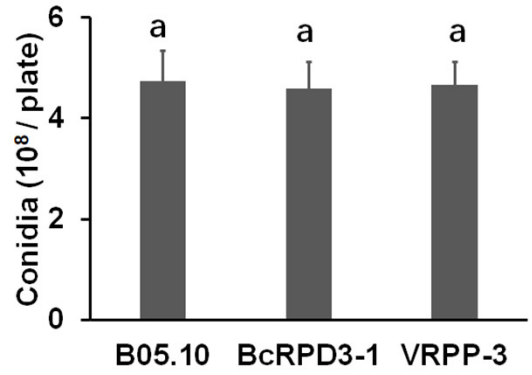

E

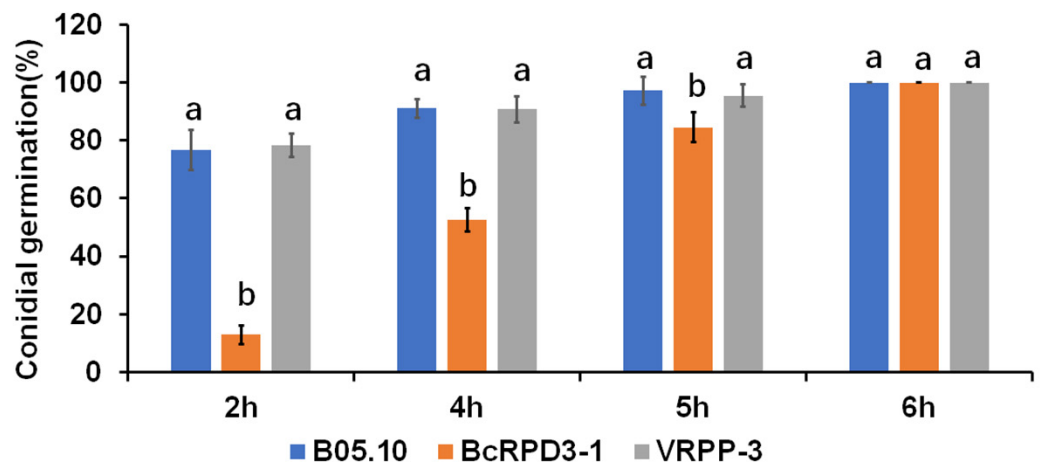

FIGURE 2 | Effects of BCRPD3 overexpression on mycelial growth, sporulation and conidial germination. The bars represent standard errors from three replicates, and statistical tests were conducted using Tukey's test for multiple comparisons. Values on the bars followed by different letters are significantly different at $p<0.05$ (A) Mycelial growth of B05.10, BcRPD3-1 and VRPP-3 strains on PDA plates after 3 days of cultivation. (B) Quantification of colony diameter of the indicated strains grown on PDA plates for 3 days. (C) Conidiation of different strains on PDA after 10 days of cultivation. (D) Quantification of conidia produced by the indicated strains on PDA plates (diameter, $4 \mathrm{~cm}$ ). (E) Quantification of the conidial germination of the indicated strains during a time course (6 h) of germination in PDB on glass slides.

leads to altered and co-regulated expression of biosynthetic gene cluster genes (Shwab et al., 2007; Pfannenstiel et al., 2018; Pidroni et al., 2018).

For the up-regulated genes, GO analysis demonstrated that they were involved in iron ion binding, oxidoreductase activity, heme binding, tetrapyrrole binding, and cofactor binding (Supplementary Figure S3A), which exhibiting a prominent characteristic of binding function. Heatmap and qPCR validation of the most significantly up-regulated genes are shown in Supplementary Figures S3B,C. Further KEGG pathway enrichment of differentially expressed genes showed that they were involved in multiple nutrients metabolisms, such as pentose, sucrose, galactose, tyrosine, and histidine (Figure 4F), indicating BcRPD3 may play a role in regulating fungal vegetative growth and development.

\section{Overexpression of BcRPD3 Results in Increased BcRPD3 and Decreased H3Kac Levels on Promoters of Pathogenicity-Related Genes}

BcRPD3 significantly affected the acetylation level of H3Kac (Figure 1D), and overexpression of BcRPD3 dramatically reduced the expression levels of 9 pathogenicity related genes (Figure 4D). To investigate whether BcRPD3 directly regulated these genes, a selective chromatin immunoprecipitation (ChIP)qPCR assay using anti-GFP was used to cross-link DNA target 
A

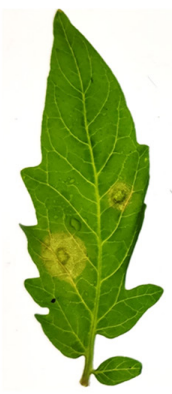

B05.10 | BcRPD3-1
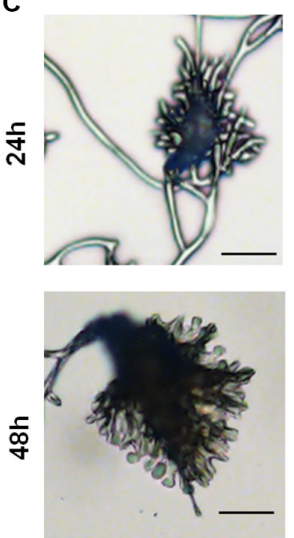

B05.10

E

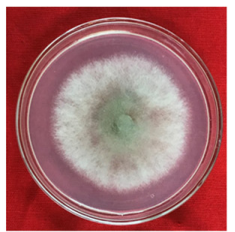

B05.10

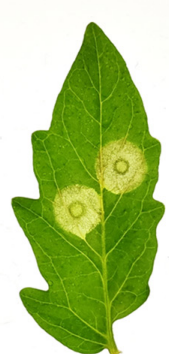

B05.10 | VRPP-3 BCRPD3-1 | VRPP-3
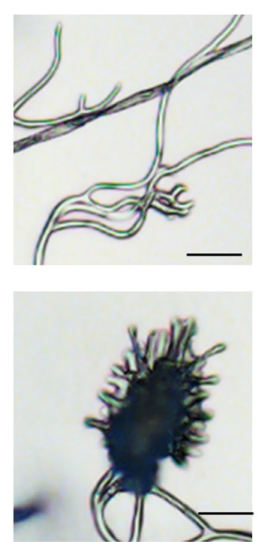

BcRPD3-1

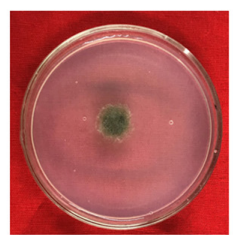

BcRPD3-1
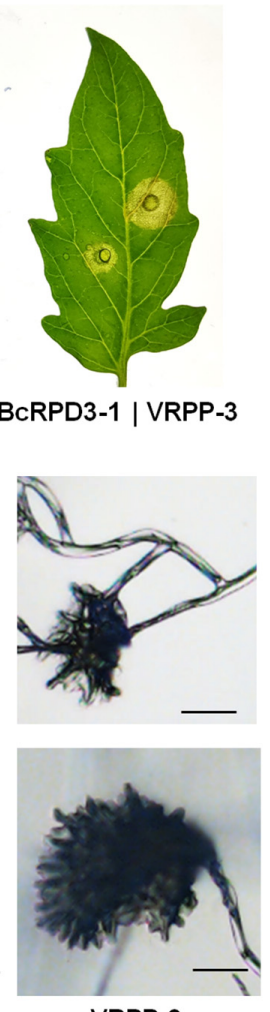

VRPP-3

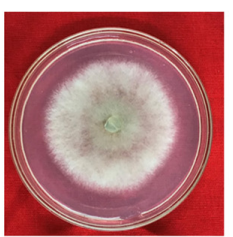

VRPP-3
B

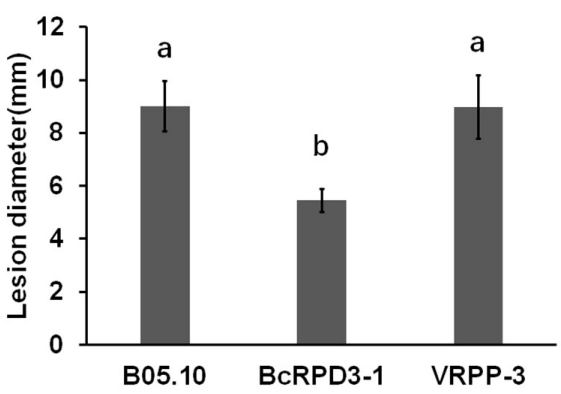

D

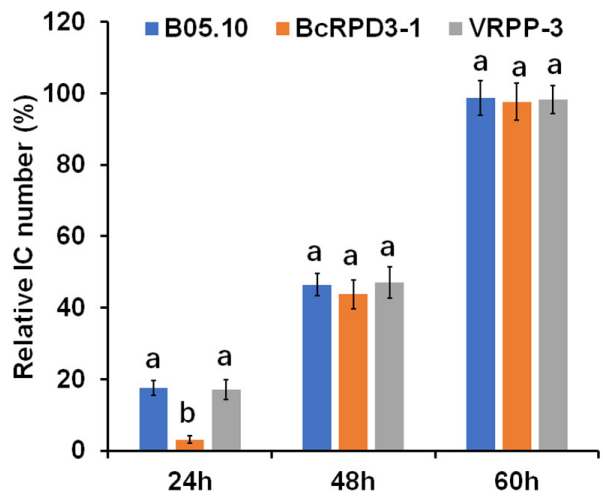

$\mathbf{F}$

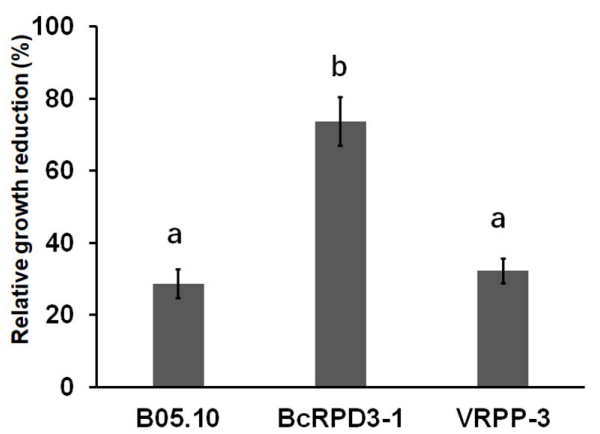

FIGURE 3 | Impact of BCRPD3 overexpression on virulence, infection cushion formation and oxidative stress response. The bars represent standard errors from five replicates, and statistical tests were conducted using Tukey's test for multiple comparisons. Values on the bars followed by different letters are significantly different at $p<0.05$. (A) Pathogenicity on tomato leaves after 3 days of incubation. (B) Quantification of disease lesions on tomato leaves 3 days after inoculation. (C) Infection cushion formation of the indicated strains after 24 and $48 \mathrm{~h}$ of incubation on glass slides. Bars $=20 \mu \mathrm{m}$. (D) Quantification of infection cushion (IC) numbers produced by the indicated strains over a time course. (E) Sensitivity of the indicated strains after cultivation on PDA plates supplemented with $0.2 \% \mathrm{H}_{2} \mathrm{O}_{2}$ for 3 days. (F) Relative growth reduction of the indicated strains which represented the reduction of colony diameters between normal and stress conditions normalized to colony diameters in normal condition.

fragments that bind BcRPD3 in vivo. Primers in promoter regions near putative TSS (transcription start site) were designed to evaluate enrichment levels of BcRPD3 in the 9 pathogenicity related genes. The results showed that these regions were highly enriched by BcRPD3 in BcRPD3-1 strain compared with wild type strain containing GFP alone (Figure 5A). ACTIN was used as a negative reference gene, and the ACTIN sequence was not enriched (Figure 5A).

To test whether these promoter regions were also $\mathrm{H} 3 \mathrm{Kac}$ locations in genomic DNA, we further performed ChIP using anti-H3Kac antibody followed by qPCR. As shown in Figure 5B, these regions were also enriched by $\mathrm{H} 3 \mathrm{Kac}$ in wild type compared with BcRPD3-1 strain, without enrichment in promoter region of ACTIN (Figure 5B). These observations demonstrated that BcRPD3 and H3Kac could enrich the promoter regions of 9 pathogenicity related genes, indicating that BcRPD3 and $\mathrm{H} 3 \mathrm{Kac}$ participated in gene transcriptional regulation in $B$. cinerea.

\section{DISCUSSION}

RPD3 and its homologs (HDAC1-3) are widely conserved across different organisms. The roles of HDAC1-3 and yeast RPD3 have been well investigated in human diseases such as cancer 
A

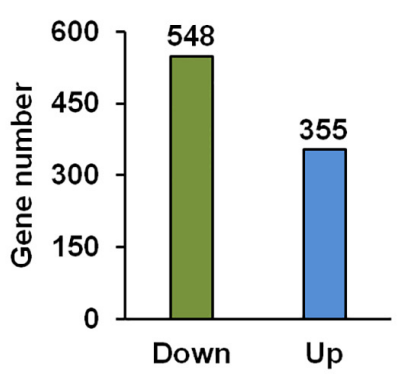

B

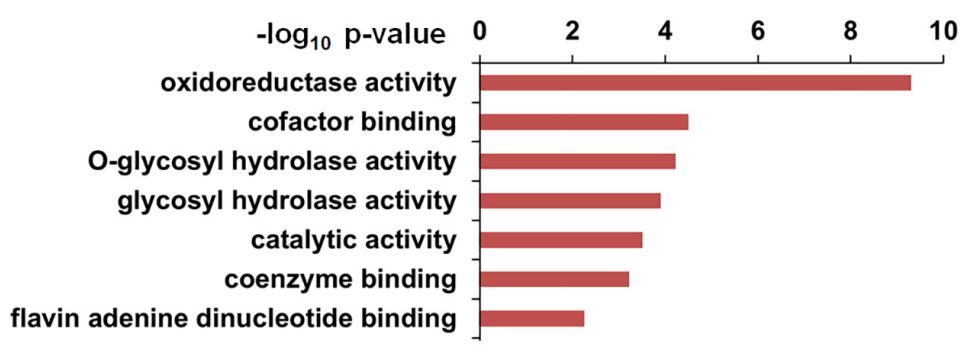

D

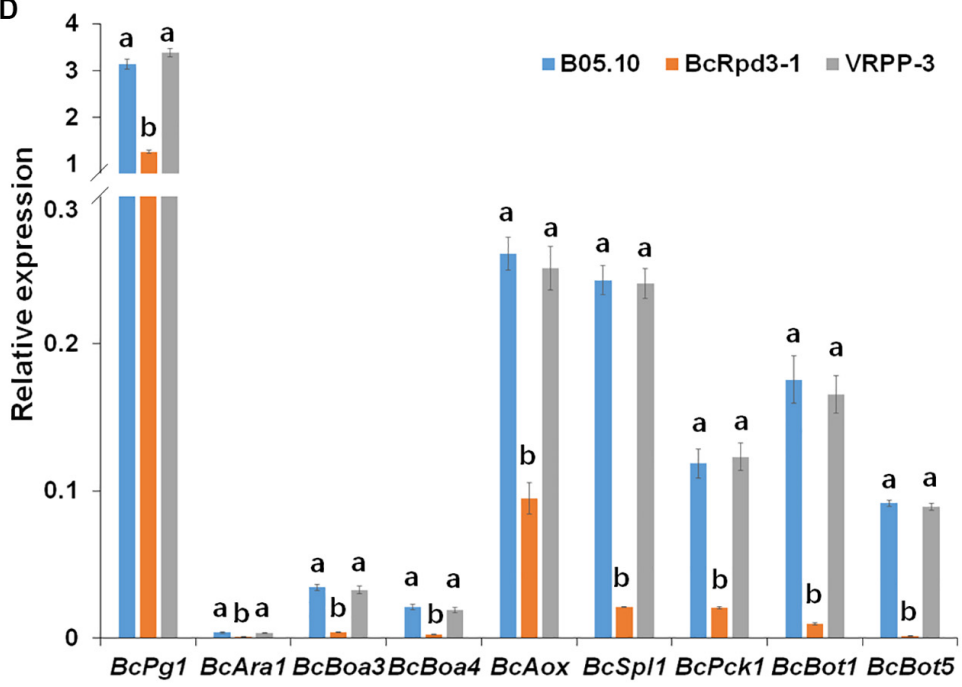

$\mathbf{F}$
E

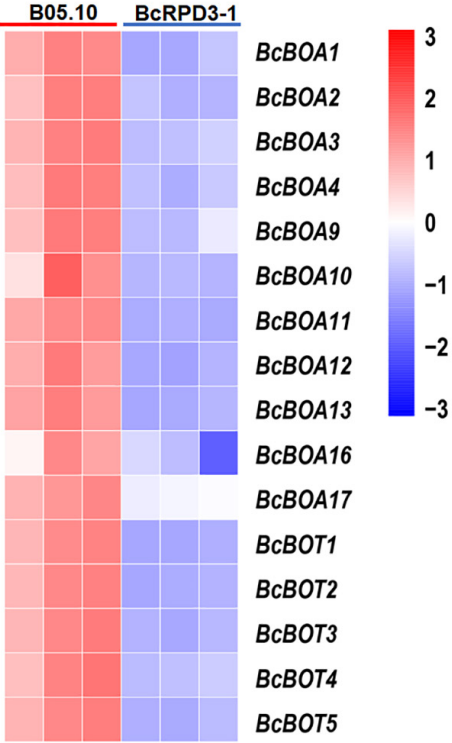

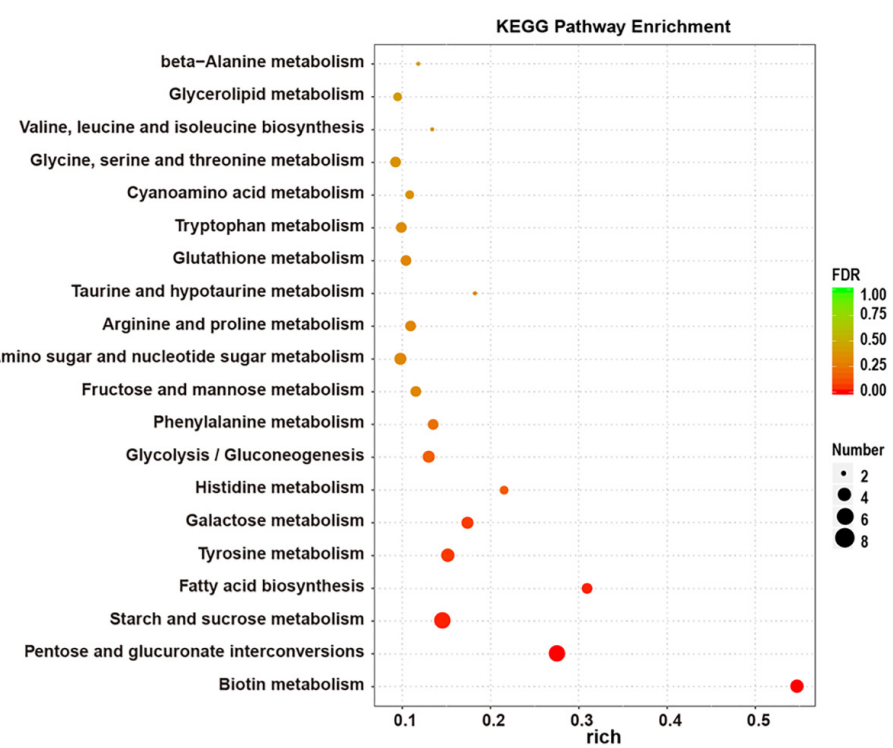

FIGURE 4 | RNA-Seq Analysis of BCRPD3 overexpression strain. (A) Numbers of up- and down regulated genes ( $P$-value $<0.05$, fold change $>2$ ) in BcRPD3-1 strain compared with wild type B05.10. (B) Distribution of functional classification of downregulated genes in BcRPD3-1. Histograms indicate $P$-values of the enriched functional categories. (C) RNA-seq analysis of 9 downregulated genes involved in enzymatic activity. Differential expression in three biological replicates is illustrated using a heat map with colored squares indicating the range of expression levels referred to log2 FPKM value. (D) qRT-PCR validation of downregulated genes. Expression levels were normalized to $\beta$-tubulin gene. The bars represent standard errors from three replicates, and statistical tests were conducted using Tukey's test for multiple comparisons. Values on the bars followed by different letters are significantly different at $p<0.05$. (E) RNA-seq analysis of $B c B o a$ and $B c B o t$ family genes. Differential expression in three biological replicates is illustrated using a heat map with colored squares indicating the range of expression levels referred to log2 FPKM value. (F) KEGG pathway enrichment of differentially expressed genes in BcRPD3-1 compared with wild type. 

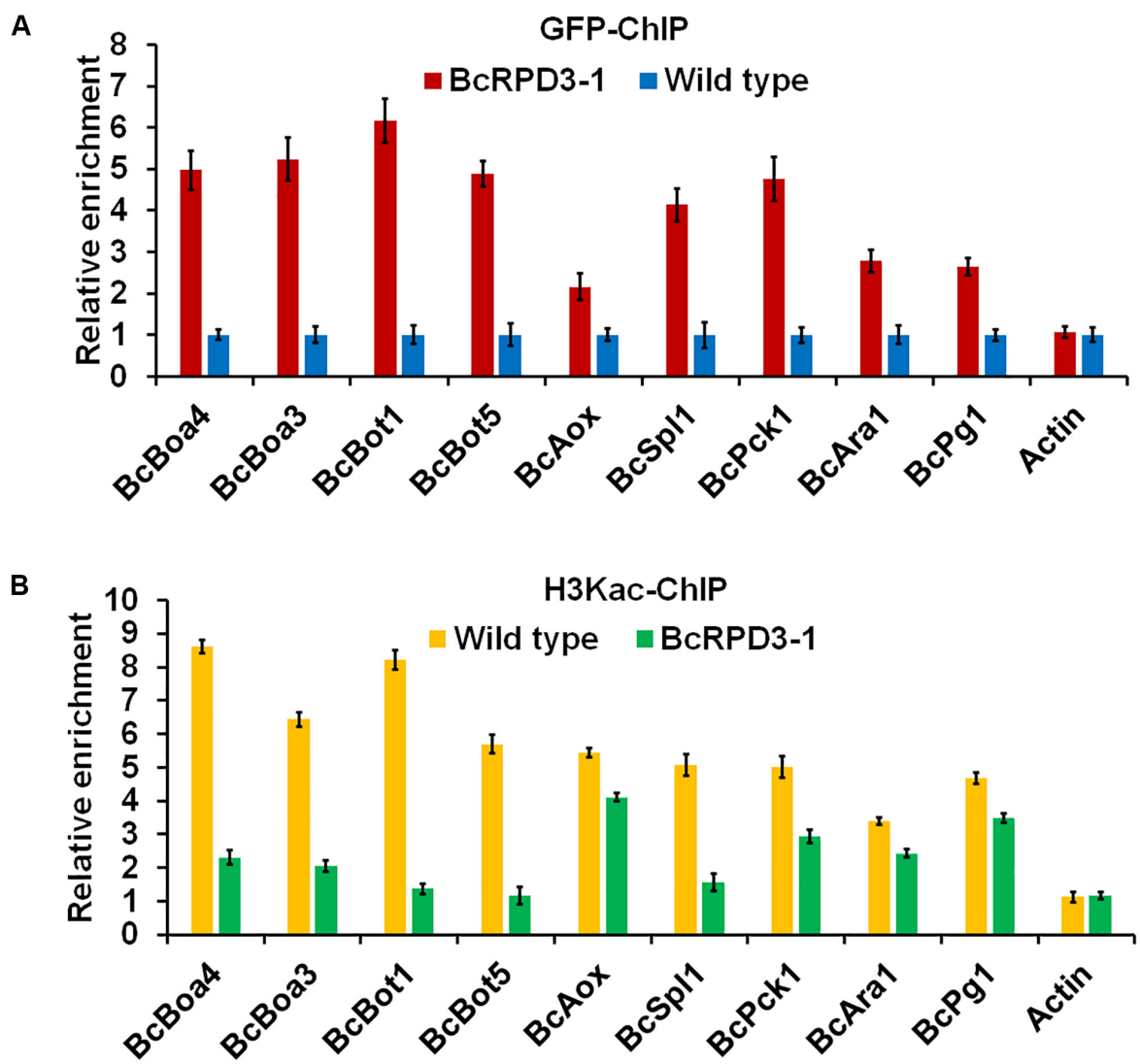

FIGURE 5 | Relative enrichment levels of immunoprecipitated promoter regions of enzymatic activity related genes. The fold enrichment was normalized to input and internal control gene ( $\beta$-tubulin-p). Data are means $\pm \mathrm{SD}(n=3)$. (A) Relative levels of the immunoprecipitated chromatin using anti-GFP antibody from the BCRPD3-1 strain and wild type containing GFP alone. (B) Relative levels of the immunoprecipitated DNA using anti-H3Kac from wild type containing GFP alone and BCRPD3-1 strain.

(Spurling et al., 2008; Hayashi et al., 2010; Müller et al., 2013), regulation of histone acetylation in yeast (Rundlett et al., 1996; Kurdistani and Grunstein, 2003; Yang and Seto, 2008), respectively. In filamentous fungi, several studies have discovered that RPD3 complexes were involved in virulence in M. oryzae, F. pseudograminearum, A. fumigatus, and so on (He et al., 2018; Bauer et al., 2019; Zhang et al., 2020). In this study, we investigated the roles of BcRPD3 as a class I HDAC in fungal growth and pathogenesis in order to bridge this knowledge gap in $B$. cinerea.

We first tried to delete $B c R P D 3$ gene to investigate its function. However, although deletion of $R P D 3$ is not lethal in $S$. cerevisiae, we failed to obtain knockout mutants after screening over 200 transformants from at least five independent transformations. In fact, many attempts of RPD3 disruption in filamentous fungi was unsuccessful, as in the case with Fusarium fujikuroi, M. oryzae, and A. nidulans (Izawa et al., 2009; Tribus et al., 2010; Studt et al., 2013; Bauer et al., 2016), suggesting that RPD3 is an essential gene in filamentous fungi. As an alternative, we generated overexpressed strains of $B c R P D 3$. Overexpression of $B C R P D 3$ dramatically decreased the global acetylation levels of $\mathrm{H} 3$ and $\mathrm{H} 4$ (Figure 1D). Together with nuclear localization and conserved domain architecture, the result strongly suggests that BcRPD3 is a bona fide histone deacetylase in B. cinerea.

Previous reports have shown that class I HDACs function as members of protein complexes in vitro. For example, in filamentous fungi, RpdA interacted with RcLS2F in A. nidulans (Bauer et al., 2020). Rpd3L-like HDAC complex in Fusarium Pseudograminearum contained multiple partners including FpDep1, FpSds3, FpSin3, FpRpd3, FpRxt3, FpCti6, FpPho23, and FpUme6 (Zhang et al., 2020). However, overexpression of RpdA in Aspergillus sp. did not cause any phenotypic effects. This has been attributed to the lack of complex partners despite overexpression of the enzyme and is in line with the observation that significant in vitro class 1 HDAC activity is only observed for enzyme complexes. From our transcriptomics data, the expression of these putative interaction partners of RPD3 did not show any significant difference between BcRPD31 and wild type, indicating that BcRPD3 acting at least in part on its own and this might indicate a different feature of BcRPD3 when compared to its orthologs of other filamentous fungal subclades.

HDACs gene family is conserved in different organisms ranging from humans to yeast (Gregoretti et al., 2004). In humans, swapping the amino acids AGG in HDAC1 with VRPP 
generated a novel HDAC1 mutant with impaired HDAC activity (Wei et al., 2017). In some cases, excessive protein expression may be toxic for organisms, resulting in damages and dysfunction. To test that possibility, we overexpressed BcRPD3-VRPP with a $\mathrm{C}$-terminal GFP in $B$. cinerea. Following western analysis using anti-H3Kac and H4Kac showed that BcRPD3-VRPP protein didn't possess any HDAC activity (Figure 1D), indicating a conserved function of VRPP mutation for class I HDACs in $B$. cinerea. Therefore, we used BcRPD3-VRPP overexpression strain (VRPP-3) as a silencing transformant and our data demonstrated that strain VRPP-3 with almost same protein expression of strain BcRPD3-1 did not show any impaired phenotype compared with wild type. It means that the changed phenotypes of strain BcRPD3-1 are caused by the reinforced HDAC function, but not excessive protein expression.

Overexpression of BcRPD3 did not affect sporulation, but diminished mycelial growth and germination slightly, pointing toward roles of BcRPD3 in regulating fungal vegetative growth and development. Our RNA-seq analysis suggests that global changes of genes involved in multiple nutrients metabolisms, such as pentose, sucrose, galactose, tyrosine, and histidine, is likely to underlie this growth defect (Figure 4F). The results suggest that the overexpression strain probably cannot properly express genes with function in the efficient transport and use of those nutrients due to excess of BcRPD3-mediated histone deacetylation.

Other than vegetative growth, we observed sharp declines in the virulence, infection cushion formation and oxidative stress response in the overexpression strain. As shown in Supplementary Figure S1, expression of BcRPD3 elevated in mycelium compared with in conidia $(0 \mathrm{~h})$ and decreased during infectious growth stages. We speculated that during vegetative growth phase, the expression of endogenous $B C R P D 3$ in $B$. cinerea was active and kept in a high level which is enough for BcRPD3 to perform its functions, so that excess BcRPD3 may only have limited effects on the vegetative growth. While during infectious stages, expression of BcRPD3 decreased dramatically and stayed in a low level. Overexpression of $B C R P D 3$ may break that native regulatory pathway and impaired the process of host infection. Therefore, we presumed that overexpression of BcRPD3 may play a more important role during the infectious stages than vegetative growth phase. Further RNA-seq analysis showed that 548 genes were significantly downregulated in BcRPD3-1 compared with wild type, including 9 pathogenicity related genes reported to act as virulence factors of $B$. cinerea (Figures $\mathbf{4 C}, \mathbf{D}$ ). Among these, BcPg1, acting as endopolygalacturonase, can converse host tissue into fungal biomass and is required for full virulence (Have et al., 1998). Deletion of phosphoenolpyruvate carboxykinase gene BcPck1 resulted in slow conidium germination, and delayed infection structure formation (Liu et al., 2018). Alternative oxidase gene $B c A o x$ is involved in regulation of virulence and oxygen stress response of $B$. cinerea (Lin et al., 2019). BcAral encoding $\alpha-1,5$-L-endo-arabinanase plays an important role during the infection of host (Nafisi et al., 2014). BcSpll is one of the most abundant proteins in the secretome of Botrytis cinerea and the knock out mutants showed decreased virulence in a variety of hosts (Frías et al., 2011). The double mutants of BcBoa and $B c B o t$ that do not produce botcinic acid or botrydial displayed markedly reduced virulence (Dalmais et al., 2011). Collectively, together with ChIP-qPCR results, BcRPD3 may directly regulate these 9 pathogenicity related genes to modulate pathogenicity of $B$. cinerea. Furthermore, as the acetylation levels of both $\mathrm{H} 3$ and $\mathrm{H} 4$ decreased in strain BcRPD3-1, future studies are necessary to explore the regulatory role of $\mathrm{H} 4 \mathrm{Kac}$ in $B c R P D 3$ overexpression strain.

It is reported that deletion of RPD3 resulted in impaired growth and virulence in several fungi (Reichmann et al., 2002; Bauer et al., 2016, 2019; Cai et al., 2018). Based on our results, enhanced BcRPD3-mediated histone deacetylation also led to diminished mycelial growth, germination, and virulence. Therefore, we speculated that maintaining an appropriate RPD3 level is critical for the survival and pathogenicity of fungal pathogens.

\section{CONCLUSION}

In conclusion, our data indicate that BcRPD3 is a conserved epigenetic component responsible for histone acetylation and the elevated HDAC activity is important for growth and pathogenicity of $B$. cinerea. Transcriptome data provide enzymatic activity related genes downregulated following BcRPD3 overexpression. The promoter regions of 9 of these genes which were reported participating fungal pathogenicity were shown to be significantly enriched by BcRPD3 and H3Kac. Further illustration of target genes would help further investigate novel control strategy for $B$. cinerea.

\section{DATA AVAILABILITY STATEMENT}

The original contributions presented in the study are publicly available. This data can be found here: https://www.ncbi.nlm.nih. gov. Bioproject ID: PRJNA627027.

\section{AUTHOR CONTRIBUTIONS}

$\mathrm{NZ}$ and WL generated the hypothesis, planned the experiments, and wrote the manuscript. NZ, ZY, and ZZ performed the experiments. All other authors provided comments on the manuscript.

\section{FUNDING}

This research was supported by the National Natural Science Foundation of China (31722044 and 31972213), the Shandong Provincial Natural Science Foundation (ZR2019BC070), the Ministry of Agriculture of China (2016ZX08009003-001), Shandong Postdoctoral Innovation Program (201903081), Taishan Scholar Construction Foundation of Shandong Province (tshw20130963), and "First Class Grassland Science Discipline" program in Shandong Province, China. 


\section{SUPPLEMENTARY MATERIAL}

The Supplementary Material for this article can be found online at: https://www.frontiersin.org/articles/10.3389/fmicb. 2020.01832/full\#supplementary-material

FIGURE S1 | Expression profile of BCRPD3 in mycelium and during host infection by the pathogen. Expression levels were normalized to BCACT1 reference gene (Liu Y. et al., 2019). Data are means \pm SD $(n=3)$. The double asterisks represent significant differences at $p<0.01$ (Student's t test).

FIGURE S2 | (A) Diagram illustrating alignment of representative class I HDAC (HDAC1) and class II HDAC (HDAC6) in human with BcRPD3 and BcHda1 in the conserved regions containing AGG/NRPP, respectively. (B) qRT-PCR validation of $B C R P D 3$ gene overexpression in different strains. Expression levels were normalized to $\beta$-tubulin gene. Data are means $\pm S D(n=3)$. (C) Immunoblot for the detection of BcRPD3-GFP in extracts of B05.10, three BcRPD3-GFP

\section{REFERENCES}

Bauer, I., Gross, S., Merschak, P., Kremser, L., Karahoda, B., Bayram, O. Z., et al. (2020). RcLS2F - A novel fungal class 1 KDAC Co-repressor complex in Aspergillus nidulans. Front. Microbiol. 11:43. doi: 10.3389/fmicb.2020. 00043

Bauer, I., Misslinger, M., Shadkchan, Y., Dietl, A. M., Petzer, V., Orasch, T., et al. (2019). The lysine deacetylase RpdA is essential for virulence in Aspergillus fumigatus. Front. Microbiol. 10:2773. doi: 10.3389/fmicb.2019.02773

Bauer, I., Varadarajan, D., Pidroni, A., Gross, S., Vergeiner, S., Faber, B., et al. (2016). A class 1 histone deacetylase with potential as an antifungal target. mBio 7:e00831-16. doi: 10.1128/mBio.00831-16

Bernstein, B. E., Tong, J. K., and Schreiber, S. L. (2000). Genomewide studies of histone deacetylase function in yeast. Proc. Natl. Acad. Sci. U.S.A. 97, 13708-13713. doi: 10.1073/pnas.250477697

Cai, Q., Wang, Z. K., Shao, W., Ying, S. H., and Feng, M. G. (2018). Essential role of Rpd3-dependent lysine modification in the growth, development and virulence of Beauveria bassiana. Environ. Microbiol. 20, 1590-1606. doi: 10.1111/14622920.14100

Carrozza, M. J., Li, B., Florens, L., Suganuma, T., Swanson, S. K., Lee, K. K., et al. (2005). Histone $\mathrm{H} 3$ methylation by Set 2 directs deacetylation of coding regions by Rpd3S to suppress spurious intragenic transcription. Cell 123, 581-592. doi: $10.1016 /$ j.cell.2005.10.023

Chang, C. S., and Pillus, L. (2009). Collaboration between the essential Esa1 acetyltransferase and the Rpd3 deacetylase is mediated by H4K12 histone acetylation in Saccharomyces cerevisiae. Genetics 183, 149-160. doi: 10.1534/ genetics.109.103846

Dalmais, B., Schumacher, J., Moraga, J., Le Pecheur, P., Tudzynski, B., Collado, I. G., et al. (2011). The Botrytis cinerea phytotoxin botcinic acid requires two polyketide synthases for production and has a redundant role in virulence with botrydial. Mol. Plant Pathol. 12, 564-579. doi: 10.1111/j.1364-3703.2010. 00692.x

De Nadal, E., Zapater, M., Alepuz, P. M., Sumoy, L., Mas, G., Posas, F., et al. (2004). The MAPK Hog 1 recruits Rpd3 histone deacetylase to activate osmoresponsive genes. Nature 427, 370-374. doi: 10.1038/nature02258

Dean, R., Van Kan, J. A., Pretorius, Z. A., Hammond-Kosack, K. E., and Di Pietro, A. (2012). The Top 10 fungal pathogens in molecular plant pathology. Mol. Plant. Pathol. 13, 414-430. doi: 10.1111/j.1364-3703.2012.00822.x

Emiliani, S., Fischle, W., Van Lint, C., Al-Abed, Y., and Verdin, E. (1998). Characterization of a human RPD3 ortholog, HDAC3. Proc. Natl. Acad. Sci. U.S.A. 95, 2795-2800. doi: 10.1073/pnas.95.6.2795

Frías, M., González, C., and Brito, N. (2011). BcSpl1, a cerato-platanin family protein, contributes to Botrytis cinerea virulence and elicits the hypersensitive response in the host. New Phytol. 192, 483-495. doi: 10.2307/41320501

Graessle, S., Dangl, M., Haas, H., Mair, K., Trojer, P., Brandtner, E. M., et al. (2000). Characterization of two putative histone deacetylase genes from Aspergillus nidulans. Biochim. Biophys. Acta 1492, 120-126. doi: 10.1016/s0167-4781(00) 00093-2 transformants and three BcRPD3-VRPP-GFP transformants using anti-GFP. Histone $\mathrm{H} 3$, which served as a loading control, was detected using anti-H3 antibody. (D) Statistical analysis of $\mathrm{H} 3$ and $\mathrm{H} 4$ acetylation. Data are means $\pm \mathrm{SD}$ $(n=3)$.

FIGURE S3 | (A) Distribution of functional classification of upregulated genes in BcRPD3-1. Histograms indicate $P$-values of the enriched functional categories. (B) RNA-seq analysis of 6 most significantly upregulated genes. Differential expression in three biological replicates is illustrated using a heat map with colored squares indicating the range of expression levels referred to log2 FPKM value. (C) qRT-PCR validation of downregulated genes. Expression levels were normalized to $\beta$-tubulin gene. Data are means $\pm \mathrm{SD}$ $(n=3)$.

\section{TABLE S1 | Primers used in the study.}

TABLE S2 | Mapping statistics of the RNA-seq data.

TABLE S3 | The RNA-seq analysis data of gene expression.

Gregoretti, I., Lee, Y.-M., and Goodson, H. V. (2004). Molecular evolution of the histone deacetylase family: functional implications of phylogenetic analysis. J. Mol. Biol. 338, 17-31. doi: 10.1016/j.jmb.2004.02.006

Gronover, C. S., Kasulke, D., Tudzynski, P., and Tudzynski, B. (2001). The role of G protein alpha subunits in the infection process of the gray mold fungus Botrytis cinerea. Mol. Plant Microbe Interact. 14, 1293-1302. doi: 10.1094/MPMI.2001. 14.11.1293

Guan, K. L., and Xiong, Y. (2011). Regulation of intermediary metabolism by protein acetylation. Trends Biochem. Sci. 36, 108-116. doi: 10.1016/j.tibs.2010. 09.003

Have, A. T., Mulder, W., Visser, J., and Van Kan, J. A. (1998). The endopolygalacturonase gene Bcpg1 is required for full virulence of Botrytis cinerea. Mol. Plant Microbe Interact. 11, 1009-1016. doi: 10.1094/MPMI.1998. 11.10.1009

Hayashi, A., Horiuchi, A., Kikuchi, N., Hayashi, T., Fuseya, C., Suzuki, A., et al. (2010). Type-specific roles of histone deacetylase (HDAC) overexpression in ovarian carcinoma: HDAC1 enhances cell proliferation and HDAC3 stimulates cell migration with downregulation of E-cadherin. Int. J. Cancer 127, 13321346. doi: 10.1002/ijc.25151

He, M., Xu, Y., Chen, J., Luo, Y., Lv, Y., Su, J., et al. (2018). MoSnt2-dependent deacetylation of histone $\mathrm{H} 3$ mediates MoTor-dependent autophagy and plant infection by the rice blast fungus Magnaporthe oryzae. Autophagy 14, 15431561. doi: 10.1080/15548627.2018.1458171

Imai, S., and Guarente, L. (2010). Ten years of NAD-dependent SIR2 family deacetylases: implications for metabolic diseases. Trends Pharmacol. Sci. 31, 212-220. doi: 10.1016/j.tips.2010.02.003

Izawa, M., Takekawa, O., Arie, T., Teraoka, T., Yoshida, M., Kumura, M., et al. (2009). Inhibition of histone deacetylase causes reduction of appressorium formation in the rice blast fungus Magnaporthe oryzae. J. Gen. Appl. Microbiol. 55, 489-498. doi: 10.2323/jgam.55.489

Jeon, J., Kwon, S., and Lee, Y.-H. (2014). Histone acetylation in fungal pathogens of plants. Plant Pathology J. 30, 1-9. doi: 10.5423/PPJ.RW.01.2014.0003

Kurdistani, S. K., and Grunstein, M. (2003). Histone acetylation and deacetylation in yeast. Nat. Rev. Mol. Cell Biol. 4, 276-284. doi: 10.1038/nrm1075

Lechner, T., Carrozza, M. J., Yu, Y., Grant, P. A., Eberharter, A., Vannier, D., et al. (2000). Sds3 (suppressor of defective silencing 3) is an integral component of the yeast Sin3. Rpd3 histone deacetylase complex and is required for histone deacetylase activity. J. Biol. Chem. 275, 40961-40966. doi: 10.1074/jbc. M005730200

Lee, K. K., and Workman, J. L. (2007). Histone acetyltransferase complexes: one size doesn't fit all. Nat. Rev. Mol. Cell Biol. 8, 284-295. doi: 10.1038/nrm2145

Leroch, M., Mernke, D., Koppenhoefer, D., Schneider, P., Mosbach, A., Doehlemann, G., et al. (2011). Living colors in the gray mold pathogen Botrytis cinerea: codon-optimized genes encoding green fluorescent protein and mCherry, which exhibit bright fluorescence. Appl. Environ. Microbiol. 77, 2887-2897. doi: 10.1128/aem.02644-10

Lin, Z., Wu, J., Jamieson, P. A., and Zhang, C. (2019). Alternative oxidase is involved in the pathogenicity, development, and oxygen stress response of 
Botrytis cinerea. Phytopathology 109, 1679-1688. doi: 10.1094/PHYTO-01-190012-R

Liu, J. K., Chang, H. W., Liu, Y., Qin, Y., Ding, Y. H., Wang, L., et al. (2018). The key gluconeogenic gene PCK1 is crucial for virulence of Botrytis cinerea via initiating its conidial germination and host penetration. Environ. Microbiol. 20, 1794-1814. doi: 10.1111/1462-2920.14112

Liu, Y., Liu, J. K., Li, G. H., Zhang, M. Z., Zhang, Y. Y., Wang, Y. Y., et al. (2019). A novel Botrytis cinerea-specific gene BcHBF1 enhances virulence of the grey mould fungus via promoting host penetration and invasive hyphal development. Mol. Plant. Pathol. 20, 731-747. doi: 10.1111/mpp.12788

Liu, Z., Jian, Y., Chen, Y., Kistler, H. C., He, P., Ma, Z., et al. (2019). A phosphorylated transcription factor regulates sterol biosynthesis in Fusarium graminearum. Nat. Commun. 10:1228. doi: 10.1038/s41467-01909145-6

Müller, B. M., Jana, L., Kasajima, A., Lehmann, A., Prinzler, J., Budczies, J., et al. (2013). Differential expression of histone deacetylases HDAC1, 2 and 3 in human breast cancer-overexpression of HDAC2 and HDAC3 is associated with clinicopathological indicators of disease progression. BMC Cancer 13:215. doi: 10.1186/1471-2407-13-215

Nafisi, M., Stranne, M., Zhang, L., Van Kan, J. A., and Sakuragi, Y. (2014). The endo-arabinanase BcAral is a novel host-specific virulence factor of the necrotic fungal phytopathogen Botrytis cinerea. Mol. Plant Microbe. Interact. 27, 781-792. doi: 10.1094/MPMI-02-14-0036-R

Pfannenstiel, B. T., Greco, C., Sukowaty, A. T., and Keller, N. P. (2018). The epigenetic reader $\mathrm{SntB}$ regulates secondary metabolism, development and global histone modifications in Aspergillus flavus. Fungal Genet. Biol. 120, 9-18. doi: 10.1016/j.fgb.2018.08.004

Pidroni, A., Faber, B., Brosch, G., Bauer, I., and Graessle, S. (2018). A class 1 histone deacetylase as major regulator of secondary metabolite production in Aspergillus nidulans. Front. Microbiol. 9:2212. doi: 10.3389/fmicb.2018.02212

Raudvere, U., Kolberg, L., Kuzmin, I., Arak, T., and Adler, P. (2019). g:Profiler: a web server for functional enrichment analysis and conversions of gene lists (2019 update). Nucleic Acids Res. 47, W191-W198. doi: 10.1093/nar/gkz369

Reichmann, M., Jamnischek, A., Weinzierl, G., Ladendorf, O., and Huber, S. (2002). The histone deacetylase Hdal from Ustilago maydis is essential for teliospore development. Mol. Microbiol. 46, 1169-1182. doi: 10.1046/j.1365-2958.2002. 03238.x

Rodenburg, S. Y. A., Terhem, R. B., Veloso, J., Stassen, J. H. M., and Van Kan, J. A. L. (2018). Functional analysis of mating type genes and transcriptome analysis during fruiting body development of Botrytis cinerea. mBio 9:e01939-17. doi: 10.1128/mBio.01939- 17

Rundlett, S. E., Carmen, A. A., Kobayashi, R., Bavykin, S., Turner, B. M., and Grunstein, M. (1996). HDA1 and RPD3 are members of distinct yeast histone deacetylase complexes that regulate silencing and transcription. Proc. Natl. Acad. Sci. U.S.A. 93, 14503-14508. doi: 10.1073/pnas.93.25.14503

Rundlett, S. E., Carmen, A. A., Suka, N., Turner, B. M., and Grunstein, M. (1998). Transcriptional repression by UME6 involves deacetylation of lysine 5 of histone H4 by RPD3. Nature 392, 831-835. doi: 10.1038/33952

Sabet, N., Volo, S., Yu, C., Madigan, J. P., and Morse, R. H. (2004). Genome-wide analysis of the relationship between transcriptional regulation by Rpd $3 p$ and the histone $\mathrm{H} 3$ and $\mathrm{H} 4$ amino termini in budding yeast. Mol. Cell. Biol. 24, 8823-8833. doi: $10.1128 / \mathrm{mcb} .24 .20 .8823-8833.2004$

Sharma, V. M., Tomar, R. S., Dempsey, A. E., and Reese, J. C. (2007). Histone deacetylases RPD3 and HOS2 regulate the transcriptional activation of DNA damage-inducible genes. Mol. Cell. Biol. 27, 3199-3210. doi: 10.1128/MCB. 02311-06

Shwab, E. K., Bok, J. W., Tribus, M., Galehr, J., and Graessle, S. (2007). Histone deacetylase activity regulates chemical diversity in Aspergillus. Eukaryot. Cell 6, 1656-1664. doi: 10.1128/ec.00186-07

Spurling, C. C., Godman, C. A., Noonan, E. J., Rasmussen, T. P., Rosenberg, D. W., Giardina, C., et al. (2008). HDAC3 overexpression and colon cancer cell proliferation and differentiation. Mol. Carcinog. 47, 137-147. doi: 10.1002/mc. 20373

Studt, L., Schmidt, F. J., Jahn, L., Sieber, C. M., Connolly, L. R., Niehaus, E. M., et al. (2013). Two histone deacetylases, FfHda1 and FfHda2, are important for Fusarium fujikuroi secondary metabolism and virulence. Appl. Environ. Microbiol. 79, 7719-7734. doi: 10.1128/aem.01557-13

Torreblanca, J. N., Stumpferl, S., and Basse, C. W. (2003). Histone deacetylase Hdal acts as repressor of the Ustilago maydis biotrophic marker gene mig1. Fungal Genet. Biol. 38, 22-32. doi: 10.1016/\$1087-1845(02)00 $505-4$

Tribus, M., Bauer, I., Galehr, J., Rieser, G., Trojer, P., and Brosch, G. (2010). A novel motif in fungal class 1 histone deacetylases is essential for growth and development of Aspergillus. Mol. Biol. Cell 21, 345-353. doi: 10.1091/mbc.e0908-0750

Trojer, P., Brandtner, E. M., Brosch, G., Loidl, P., Galehr, J., Linzmaier, R., et al. (2003). Histone deacetylases in fungi: novel members, new facts. Nucleic Acids Res. 31, 3971-3981. doi: 10.1093/nar/gkg473

Vogelauer, M., Rubbi, L., Lucas, I., Brewer, B. J., and Grunstein, M. (2002). Histone acetylation regulates the time of replication origin firing. Mol. Cell 10, 1223-1233. doi: 10.1016/s1097-2765(02)00702-5

Wei, W., Liu, X., Chen, J., Gao, S., Lu, L., Zhang, H., et al. (2017). Class I histone deacetylases are major histone decrotonylases: evidence for critical and broad function of histone crotonylation in transcription. Cell Res. 27, 898-915. doi: $10.1038 / \mathrm{cr} .2017 .68$

Yang, Q., Zhang, J., Hu, J., Wang, X., Lv, B., Liang, W., et al. (2018). Involvement of BcYak1 in the regulation of vegetative differentiation and adaptation to oxidative stress of Botrytis cinerea. Front. Microbiol. 9:281. doi: 10.3389/fmicb. 2018.00281

Yang, X. J., and Seto, E. (2008). The Rpd3/Hdal family of lysine deacetylases: from bacteria and yeast to mice and men. Nat. Rev. Mol. Cell Biol. 9, 206-218. doi: $10.1038 / \mathrm{nrm} 2346$

Zhang, Y., Wang, L., Liang, S., Zhang, P., and Kang, R. (2020). FpDep1, a component of $\mathrm{Rpd} 3 \mathrm{~L}$ histone deacetylase complex, is important for vegetative development, ROS accumulation, and pathogenesis in Fusarium pseudograminearum. Fungal Genet. Biol. 135:103299. doi: 10.1016/j.fgb.2019. 103299

Conflict of Interest: The authors declare that the research was conducted in the absence of any commercial or financial relationships that could be construed as a potential conflict of interest.

Copyright (c) 2020 Zhang, Yang, Zhang and Liang. This is an open-access article distributed under the terms of the Creative Commons Attribution License (CC BY). The use, distribution or reproduction in other forums is permitted, provided the original author(s) and the copyright owner(s) are credited and that the original publication in this journal is cited, in accordance with accepted academic practice. No use, distribution or reproduction is permitted which does not comply with these terms. 